Canum 2000: Actes du 32e Congrès national d'analyse numérique

A. Blouza, I. Danaila, P. Joly, S.M. Kaber, B. Lucquin, F. Murat \& M. Postel, Éditeurs

ESAIM: Proceedings, Vol. 11, 2002, 141-152

http://www.emath.fr/Maths/Proc/Vol.11/

(C)2002, Société de Mathématiques Appliquées et Industrielles, EDP Sciences

\title{
Modélisation du transport des charges électriques dans les semiconducteurs
}

\author{
Frédéric Poupaud
}

\begin{abstract}
Résumé. La physique des semiconducteurs est un domaine tràs riche en problàmes de modélisation et de mathématiques. Selon l'échelle considérée : microscopique, mésoscopique ou macroscopique, la modélisation du transport des charges requiert une description quantique, cinétique ou fluide. Les différents systàmes d'équations aux dérivées partielles correspondant sont décrit dans cet article. L'analyse asymptotique permet en outre de comprendre les liens profonds qui les lient. En particulier le passage des équations de la mécanique quantique aux équations cinétiques grâce aux mesures de Wigner est détaillé.
\end{abstract}

Mots clés. Semiconducteurs, limites semi-classiques, limites fluides, mesures de Wigner.

\begin{abstract}
A lot of modelling issues and mathematical problems come from semiconductors physics. Depending on the scale (microscopic, mesoscopic or macroscopic) the modelling of charge transport phenomena require a quantum, kinetic or fluid description. The corresponding P.D.E. systems are described in this paper. Moreover asymptotics analysis allow to understand the deep links and analogies between them. In particular the semi-classical limit of quantum mechanics via Wigner measures is detailed.
\end{abstract}

Key words. Semiconductors, semi-classical limits, fluid limits, Wigner measures.

AMS subject classification. 41A60, 35Q20, 76P05

\section{Introduction}

Les circuits intégrés qui sont à la base du fonctionnement des ordinateurs sont constitués de milliers de composant élémentaires. Ces composants de la taille de quelques microns sont principalement des diodes et des transistors. Les diodes ont la particularité de ne laisser passer le courant électrique que dans un sens, les transistors agissent comme des interrupteurs. A partir de ces composants élémentaires il est possible de construire des circuits logiques complexes.

Les composants ont été gravés par un procédé particulier, l'épitaxie, sur un cristal semiconducteur constitué par exemple de silicium ou d'arsenuire de gallium. L'épitaxie consiste, par jets moléculaires dans une chambre à vide, à introduire dans un cristal pur des impuretés qui permettent de controler la quantité de charges électriques libres. Ce sont ces charges électriques qui assurent l'existence d'un courant dans le composant. Un cristal semiconducteur est dit dopé si il 
contient des impuretés. Un cristal semiconducteur non dopé se comporte comme un isolant alors qu'un cristal tràs dopé se comporte comme un conducteur.

Au niveau de la conception des circuits logiques et du circuit intégré dans son ensemble, les composant élémentaires (diodes, transistors) sont modélisés par leurs caractéristiques. La caractéristique d'un composant est la courbe donnant le courant circulant dans le dispositif en fonction des potentiels qui lui sont appliqués. Cette caractéristique peut être déterminée par des mesures directes. Cependant la mise au point de nouveaux dispositifs avec des nouveaux matériaux est chàre et longue. Il faut ainsi plusieurs mois pour lancer la fabrication d'une chaine de circuits imprimés en chambre à épitaxie.

Il est donc nécessaire d'avoir des outils de modélisation qui permettent de simuler efficacement et rapidement de nouveaux composants. La simulation numérique donne in fine la caractéristique des dispositifs. Elle permet en outre une étude précise de la physique du transport des charges à l'intérieur des composants. Cette étude est tràs difficile voir impossible à partir de mesures expérimentales.

Le but de cet article est de présenter quelques aspects mathématiques des modàles employés dans ce domaine.

\section{Mécanique quantique et structure de bande}

Le niveau le plus fin de modélisation est celui de la mécanique quantique. Les électrons supposés indépendant les uns des autres sont représentés par leurs fonctions d'ondes, $\psi(t, x) \in \mathbb{C}$, qui dépendent du temps $t \in \mathbb{R}$ et de la position $x \in \mathbb{R}^{3}$. Dans un cristal parfait ces fonctions d'ondes sont solutions de l'équation de Schrödinger :

$$
i \hbar \frac{\partial}{\partial t} \psi=H(\psi)=-\frac{\hbar^{2}}{2 m} \Delta \psi+q V(x) \psi .
$$

Les constantes physiques $q, m, \hbar$ sont respectivement la charge, la masse élémentaire de l'électron et la constante de Planck réduite. Le potentiel électrique $V$ est dû aux atomes ionisés du cristal. Il est périodique. Si $\Gamma$ est le réseau direct du cristal, on a :

$$
V(x+\gamma)=V(x), \quad \gamma \in \Gamma .
$$

Une méthode usuelle de résolution de l'équation (1) est la méthode de décomposition spectrale qui consiste à diagonaliser l'hamiltonien $H$ sur une base de fonctions propres. Malheureusement en domaine non borné le spectre de $H$ n'est pas discret, [24]. Il existe cependant des sous espaces propres de dimensions infinies qui sont en somme directe dans $L^{2}\left(\mathbb{R}^{3}\right)$. Ce sont les sous espace de Floquet, chaque sous espace correspondant à un niveau de bande en langage de physique. On les obtient de la maniàre suivante. Soit $\Gamma^{\prime}$ le réseau reciproque à $\Gamma$

$$
\Gamma^{\prime}=\left\{\mu \in \mathbb{R}^{3} ; \quad \forall \gamma \in \Gamma \quad \mu . \gamma \in 2 \pi \mathbb{Z}\right\} .
$$

On note $B=\mathbb{R}^{3} / \Gamma^{\prime}$. Pour $k \in B$ on dit qu'une fonction $u$ est k-quasipériodique si elle vérifie $u(x+\gamma)=e^{i k \cdot \gamma} u(x)$ pour tout $\gamma \in \Gamma$. On remarque alors que toute fonction $\psi \in L^{2}\left(\mathbb{R}^{3}\right)$ peut se 
décomposer en fonctions k-quasipériodiques $u(., k)$ avec

$$
\psi(x)=\int_{B} u(x, k) \frac{d k}{|B|}, \quad u(x, k)=\sum_{\gamma \in \Gamma} \psi(x+\gamma) e^{-i k \cdot \gamma} .
$$

Or si $\psi$ est solution de (1) et si $V$ vérifie (2), pour $k$ fixé la fonction $u$ résoud une équation de Schrödinger identique à (1) avec des conditions de k-quasipériodicité. On note $H_{k}$ l'hamiltonien correspondant. L'avantage est que maintenant les opérateurs $H_{k}$ admettent une décomposition spectrale. On peut peut ainsi écrire $u(t, x, k)=\sum_{n=1}^{\infty} u_{n}(t, x, k)$ où les $u_{n}(t, ., k)$ sont des fonctions propres de $H_{k}$ associées aux valeurs propres $\mathcal{E}_{n}(k) \in \mathbb{R}, \mathcal{E}_{n}(k) \rightarrow \infty$ pour $n \rightarrow \infty$. L'équation de Schrödinger pour $u_{n}$ se réduit alors à

$$
i \hbar \frac{\partial}{\partial t} u_{n}(t, x, k)=\mathcal{E}_{n}(k) u_{n}(t, x, k)
$$

Grâce à (3), on obtient une décomposition sur les sous espaces de Floquet de $\psi$ :

$$
\psi(t, x)=\sum_{n=1}^{\infty} \psi_{n}(t, x), \quad \psi_{n}(x)=\int_{B} u_{n}(x, k) \frac{d k}{|B|} .
$$

Les fonctions $\psi_{n}$ appartiennent au $n^{\text {eme }}$ sous espace de Floquet. On déduit alors de (4), (5) et du développement en série de Fourier de $u_{n}$ donné par (3) avec $\psi=\psi_{n}$ que les fonctions $\psi_{n}$ résolvent :

$$
i \hbar \frac{\partial}{\partial t} \psi_{n}(t, x)=\sum_{\gamma \in \Gamma} \epsilon_{n}(\gamma) \psi_{n}(x+\gamma), \quad \operatorname{avec} \mathcal{E}_{n}(k)=\sum_{\gamma \in \Gamma} \epsilon_{n}(\gamma) e^{i k \cdot \gamma}
$$

Dans un langage plus physique on dit qu'un électron appartient à la $n^{e m e}$ bande d'énergie si sa fonction d'onde appartient au $n^{\text {eme }}$ sous-espace de Floquet. Les fonctions $k \rightarrow \mathcal{E}_{n}(k)$ sont les diagrammes de bande, $\mathcal{E}_{n}(k)$ étant l'énergie associée au vecteur d'onde $k$. Nous renvoyons pour un exposé plus détaillé de la théorie de Floquet à [24, 28].

Dans la pratique il n'est pas utile de considérer toutes les bandes d'énergie. En effet les bandes d'énergie élevée, les bandes de conduction, sont pratiquement vide et les bandes de basse énergie, les bandes de valences, correspondent à des électrons liés aux atomes et sont pleines. Elles ne participent donc pas à la conduction électrique. Les seules bandes qui jouent un role sont la premiàre bande de conduction et la derniàre bande de valence correspondant aux diagrammes de bandes $\mathcal{E}_{v}(k), \mathcal{E}_{c}(k)$.

\section{Dopage des semiconducteurs}

Dans un cristal semiconducteur pur la bande de valence est pleine, la bande de conduction est vide, le matériau se comporte comme un isolant. Par contre si le cristal contient des impuretés de type donneur, comme par exemple des atomes de phosphore de symbole chimique $P$, ces impuretés ont tendance à s'ionisées pour donner un électron qui va peupler la bande de conduction 
du semiconducteur. On a ainsi création d'une charge négative mobile, l'électron de la bande de conduction, et d'une charge positive fixe, l'ion du donneur, dans notre exemple $P^{+}$.

Le semiconducteur qui doit alors sa conductivité à des électrons libres de la bande de conduction est dit de type $\mathrm{N}$.

Réciproquement, si le cristal contient des impuretés de type accepteur, comme des atomes d'indium, In, elles ont tendance à capturer un électron de la bande de conduction. Il y a création d'une charge négative fixe due à l'ion, ici $I n^{-}$. D'autre part il y a un manque d'électron dans la bande de valence qui se comporte comme une charge positive mobile. Il est alors commode de considérer cette lacune comme une quasi-particule, c'est le concept de trou. D'un point de vue mathématique cela correspont à un changement d'inconnue simple (grossiàrement un changement de signe).

Le semiconducteur qui doit sa conductivité aux charges positives mobiles, les trous, est dit alors de type $\mathrm{P}$.

C'est la coexistence de région de type $\mathrm{P}$ et d'autre de type $\mathrm{N}$ qui permet la conception de dispositifs comme les diodes ou les transistors.

Le dopage d'un semiconducteur est, par définition, la concentration en charge des impuretés ionisées. Elle est positive dans les régions de type $\mathrm{N}$, négative dans les régions de type $\mathrm{P}$. Ce dopage est fixé lors de la fabrication du circuit intégré. Dans la suite on notera $C(x) \in \mathbb{R}, \quad x \in \mathbb{R}^{3}$, ce dopage.

D'autre part, pour des raisons de simplicité d'exposition, nous n'allons considérer à partir de maintenant que des semiconducteurs de type $\mathrm{N}$ et que la modélisation des phénomànes de transport dus aux électrons de la bande de conduction. Notons simplement que la modélisation du transport des trous est tout à fait similaire.

\section{Transport semi-classique}

Le transport d'un électron dans un cristal n'est pas identique au transport dans le vide et dépend fortement de la bande d'énergie dans lequel il évolue. Pour comprendre ce fait, il est nécessaire de retourner à la mécanique quantique et à son équivalent dans l'espace des phases introduit par E. Wigner, [27]. Pour une fonction d'onde donnée, $\psi(x) \in \mathbb{C}, \quad x \in \mathbb{R}^{3}$, E. Wigner a introduit la fonction :

$$
W(x, \xi)=\int_{\mathbb{R}^{3}} \psi\left(x+\frac{\hbar}{2} y\right) \bar{\psi}\left(x-\frac{\hbar}{2} y\right) e^{i \xi \cdot y} d y .
$$

Cette transformée de Wigner et sa limite classique quand $\hbar \rightarrow 0$ a été étudiée récemment par P.L. Lions et T. Paul, [17], et dans une optique légàrement différente par P. Gérard, [12]. Elle donne un puissant outil pour étudier le lien entre mécanique classique et quantique. Contrairement aux H-mesures de L. Tartar, [25], ou aux mesures de défaut de P. Gérard, [13], les transformées de Wigner donnent une description des oscillations pour une longueur d'onde particuliàre : $\hbar$. Une propriété fondamentale de cette transformée est que les observables de la mécanique quantique qui sont quadratiques en $\psi$ deviennent linéaires en $W$. Par exemple, la probabilité de présence

ESAIM: Proc., Vol. 11, 2002, 141-152 
d'une particule, $n(x) \in \mathbb{R}^{+}$, est donnée par :

$$
n(x)=\psi(x) \bar{\psi}(x)=\int_{\mathbb{R}^{3}} W(x, \xi) d \xi .
$$

Pour obtenir un outil similaire qui tiennent compte de la périodicité du cristal, on forme les séries de Wigner, [18] :

$$
w(x, k)=\sum_{\gamma \in \Gamma} \psi\left(x+\frac{\gamma}{2}\right) \bar{\psi}\left(x-\frac{\gamma}{2}\right) e^{-i k \cdot \gamma} .
$$

De la même façon que pour la transformée de Wigner on a :

$$
n(x)=\int_{B} w(x, k) \frac{d k}{|B|} .
$$

On a vu d'autre part dans le chapitre 2 que la fonction d'onde d'un électron de la bande de conduction vérifie une équation de type (6). On en déduit par un calcul facile mais fastidieux que la série de Wigner associée vérifie :

$$
i \hbar \frac{\partial}{\partial t} w(t, x, k)=\sum_{\gamma \in \Gamma} \epsilon_{c}(\gamma)\left[w\left(x+\frac{\gamma}{2}\right)-w\left(x-\frac{\gamma}{2}\right)\right] e^{i k \cdot \gamma}
$$

avec

$$
\mathcal{E}_{c}(k)=\sum_{\gamma \in \Gamma} \epsilon_{c}(\gamma) e^{i k \cdot \gamma}
$$

$\mathcal{E}_{c}$ étant le diagramme d'énergie de la bande de conduction. L'approximation semi-classique de l'équation de Wigner s'obtient alors en remplaçant les différences finies de (9) par un développement de Taylor à l'ordre 1 . On obtient finalement que $w(t, x, k) \approx f(t, x, k)$ où la fonction $f$ résout l'équation de transport :

$$
\frac{\partial}{\partial t} f(t, x, k)+v_{c}(k) \nabla_{x} f(t, x, k)=0, \quad \text { avec } \quad v_{c}(k)=\frac{1}{\hbar} \nabla_{k} \mathcal{E}_{c}(k) .
$$

Cette approximation semi-classique peut être rigoureusement justifiée, voir [12, 18]. De plus on peut démontrer que $f$ est positive, ce qui n'est pas vrai pour $w$, et que l'on a :

$$
n(t, x) \approx \int_{B} f(t, x, k) \frac{d k}{|B|} .
$$

La fonction $v_{c}(k)$ s'interpràte comme étant la vitesse d'un électron de la bande de conduction de vecteur d'onde $k$. On pourra consulter aussi l'article [14] pour une étude exhaustive des propriétés des mesures et des séries de Wigner ainsi que pour leurs applications à l'étude asymptotique des E.D.P.

Quand on rajoute un champ électrique extérieur $E(t, x)=-\nabla_{x} U(t, x)$, un développement asymptotique formel de l'équation de Schrödinger

$$
i \hbar \frac{\partial}{\partial t} \psi=H(\psi)=-\frac{\hbar^{2}}{2 m} \Delta \psi+q V(x) \psi+q U(t, x) \psi
$$


conduit à une équation de Vlasov :

$$
\frac{\partial}{\partial t} f(t, x, k)+v_{c}(k) \cdot \nabla_{x} f(t, x, k)-\frac{q}{\hbar} E(t, x) \cdot \nabla_{k} f(t, x, k)=0 .
$$

Le problàme de la justification rigoureuse de cette équation est plus délicat que la justification de (10).

La dynamique non Newtonienne relative à (13) est donnée par :

$$
\dot{X}=v_{c}(K), \quad \dot{K}=-\frac{q}{\hbar} E(t, X) .
$$

Ce résultat indique que les forces agissent sur les vecteurs d'onde et non pas sur l'accélération. Les solutions de (13) sont les fonctions constantes le long des trajectoires de (14).

Pour comprendre la difficulté de la justification mathématique de (13), il suffit de considérer le cas où les particules sont soumises à une accélération constante $\left(E=C^{t e}\right)$. Comme $K$ vit dans un tore le mouvement des particules est alors périodique en temps et leur déplacement moyen est nul! Ce phénomàne paradoxal est difficile à observer expérimentalement mais existe néanmoins : ce sont les oscillations Brillouin. Une autre façon de voir cette difficulté est de remarquer que les sous espaces de Floquet introduits précedemment ne sont plus invariant sous l'action de $l^{\prime}$ Hamiltonien $H$. Il faut en quelque sorte démontrer que l'interaction entre sous espaces induite par le potentiel $U$ est asymptotiquement nulle.

Dans un travail en commun avec P. Bechouche, [7] nous avons trouvé une formule portant sur les séries de Wigner qui décrit cette interaction. En particulier il apparait explicitement que l'interaction est faible excepté aux points où les bandes se coupent $\left(\mathcal{E}_{n}(k)=\mathcal{E}_{m}(k)\right.$ pour $\left.m \neq n\right)$. Grâce à cette formule et au prix de complications techniques considérables nous avons pu avec $\mathrm{P}$. Bechouche et N. Mauser, [6] traiter complètement le cas non linéaire du passage du systàme de Schrödinger Poisson vers les équations de Vlasov Poisson semi-classique. Le potentiel extérieur est alors obtenu en résolvant

$$
-\epsilon_{r} \Delta_{x} U(t, x)=C(x)-q n(t, x)
$$

où $\epsilon_{r}$ est la permitivité du milieu.

On retrouve une dynamique classique si on fait une approximation supplémentaire dite approximation de la bande parabolique. Elle s'obtient quand autour de $k=0$ on a :

$$
\mathcal{E}_{c}(k) \approx \mathcal{E}_{c}(0)+\frac{1}{2} \frac{\hbar^{2}}{m^{*}}|k|^{2}
$$

Cette formule définit en fait la constante $m^{*}$ qui a la dimension d'une masse. $m^{*}$ est appelée masse effective. Elle dépend fortement du diagramme de bande et donc du matériau considéré. Elle est en général tràs différente de la masse de l'électron dans le vide. La dynamique (14) devient alors Newtonienne avec $m^{*} \ddot{X}=-q E(t, X)$.

Il faut remarquer ici que pour avoir des composants rapides la masse effective doit être la plus petite possible. Cela explique l'intéret pour des matériaux comme l'arsenuire de galium pour lesquels cette masse effective est plus faible que dans le plus classique silicium.

La justification mathématique à partir de l'équation de Schrödinger de ces approximations de masses effectives a d'abord été donnée dans un cadre linéaire dans un travail en commun avec P. Ringhoffer, [23]. Le cas non linéaire a été traité par P. Bechouche, [5].

ESAIM: Proc., Vol. 11, 2002, 141-152 


\section{L'équation de Boltzmann des semiconducteurs}

Quand on considàre la population totale des électrons, on obtient une description statistique (ou cinétique) de sa dynamique en considérant la fonction de distribution $f(t, x, k)$ qui donne le nombre d'électrons par unité de volume de l'espace des phases au temps $t$, à la position $x$ et avec le vecteur d'onde $k$. Dans un cristal parfait, cette fonction résout l'equation de Vlasov (13), voir [18]. Elle vérifie de plus $0 \leq f \leq 1$. Cela signifie qu'au plus un électron occupe l'état quantique $(x, k)$. Cette particularité est commune à toute les particules élémentaires de la famille des Fermions. C'est le principe d'exclusion de Pauli.

Connaissant la fonction de distribution $f$, on calcule la concentration $n$, le flux $j$ et la densité d'énergie volumique $e$ en prenant des moyennes par rapport aux vecteurs d'onde. On a ainsi :

$$
\begin{array}{r}
n(t, x)=\int_{B} f(t, x, k) \frac{d k}{|B|}, \\
j(t, x)=\int_{B} v_{c}(k) f(t, x, k) \frac{d k}{|B|}, \\
e(t, x)=\int_{B} \mathcal{E}_{c}(k) f(t, x, k) \frac{d k}{|B|} .
\end{array}
$$

Ces formules sont à rapprocher de (11). Elles peuvent être justifiées à partir de la mécanique quantique, voir $[12,18]$.

En pratique l'équation (13) n'est pas satisfaisante pour la modélisation des dispositifs. En effet, elle ne tient pas compte des interactions entre les électrons et les impuretés et entre les électrons et les vibrations du cristal (les phonons). Or ce sont ces interactions qui thermalisent la population d'électrons. Ainsi dans un semiconducteur en équilibre la vitesse moyenne des électrons est nulle et la température des électrons est celle du cristal.

Au niveau cinétique, ces phénomànes sont modélisés par des opérateurs de collisions qui sont des opérateurs intégraux en la variable $k$. La fonction de distribution résout alors l'équation de Boltzmann des semiconducteurs qui s'écrit :

$$
\frac{\partial}{\partial t} f(t, x, k)+v_{c}(k) . \nabla_{x} f(t, x, k)-\frac{q}{\hbar} E(t, x) . \nabla_{k} f(t, x, k)=Q(f)(t, x, k) .
$$

On ne détaillera pas la forme de l'opérateur de collision $Q$. On notera simplement que la propriété de thermalisation se traduit, d'un point de vue mathématique, par la détermination du noyau de $Q$ :

$$
N(Q)=\left\{f=\frac{1}{1+\exp \left(\frac{q \mu(t, x)-\mathcal{E}_{c}(k)}{k_{B} T}\right)}\right\},
$$

où $k_{B}$ et $T$ sont la constante de Boltzmann et la température (fixée) du dispositif. Les fonctions du noyau sont les distributions de Fermi Dirac. La fonction $\mu$ qui a-priori est quelconque est le potentiel chimique. D'autre part, une propriété liée à la conservation du nombre de particules est que :

$$
\int_{B} Q(f) d k=0, \quad \text { pour tout } f
$$


Pour compléter l'équation (17), il reste à déterminer le champ électrique $E$. Il suit les lois habituelles de l'électrostatique $E(t, x)=-\nabla_{x} U(t, x)$ où le potentiel $U$ résout l'équation de Poisson (15). Le dopage $C$ a été défini dans le paragraphe 3 et la concentration $n$ dépend de $f$ par l'intermédiaire de (16).

Le systàme d'inconnues $f, E, U$, composé de (17) et (15) est quadratique. Une premiàre nonlinéarité est due au terme $E . \nabla_{k} f$ car $E$ dépend linéairement de $f$ au vu de (15), (16). L'autre non linéarité provenant du terme de collision. Il est cependant possible d'avoir une théorie d'existence et d'unicité des solutions, voir [21,20]. Nous renvoyons à [19] pour un exposé plus complet sur les propriétés de l'équation de Boltzmann des semiconducteurs.

\section{Limite fluide des équations de Boltzmann}

Les équations cinétiques restent difficiles à résoudre numériquement car elles font intervenir la variable supplémentaire $k$. Par exemple, la simulation d'un dispositif 2D dans l'espace réel donne lieu à une discrétisation d'un domaine de $\mathbb{R}^{5}$ (2D pour l'espace, 3D pour les vecteurs d'ondes). C'est pourquoi dans la pratique on cherche dàs que possible à utiliser des modàles fluides, c'est à dire des modàles qui ne font intervenir que les variables temps et espace.

La validité des modàles fluides repose toujours sur une hypothàse d'équilibre local qui revient à spécifier la dépendance en la variable $k$ de la fonction de distribution $f$. Il est alors possible de trouver des équations sur les quantités macroscopiques $n, j, e$, définies en (16).

Dans certain cas il est possible de valider cette hypothàse d'équilibre local. Ainsi pour obtenir les modàles dits de dérive-diffusion, on fait un changement d'échelle dans les équations (17), (15) qui fait apparaitre un petit paramàtre $\epsilon$. Ce paramàtre est une mesure du libre parcours moyen (le parcours moyen des électrons entre deux collisions). Les équations deviennent :

(20) $\epsilon^{2} \frac{\partial}{\partial t} f^{\epsilon}(t, x, k)+\epsilon\left(v_{c}(k) . \nabla_{x} f^{\epsilon}(t, x, k)+\nabla_{x} U^{\epsilon}(t, x) . \nabla_{k} f^{\epsilon}(t, x, k)\right)=Q\left(f^{\epsilon}\right)(t, x, k)$,

$$
-\Delta U^{\epsilon}(t, x)=C(x)-n^{\epsilon}(t, x), \quad n^{\epsilon}(t, x)=\int_{B} f^{\epsilon}(t, x, k) \frac{d k}{|B|} .
$$

Le problème est alors de déterminer les limites de $f^{\epsilon}$ et de $U^{\epsilon}$ quand $\epsilon \rightarrow 0$.

Formellement on obtient que la limite $f$ de $f^{\epsilon}$ vérifie $Q(f)=0$. Par conséquent $f$ est une fonction de Fermi Dirac (18). Le passage à la limite dans (21) est immédiat et l'on obtient que la limite $U$ de $U^{\epsilon}$ résout :

$$
-\Delta U(t, x)=C(x)-N(\mu(t, x)),
$$

la fonction $N(\mu)$ donnant la concentration de la fonction de Fermi Dirac de potentiel chimique $\mu$. Il reste à déterminer le potentiel chimique $\mu$. Avec l'ansatz $f^{\epsilon}=f+\epsilon h$, en utilisant la propriété (19) et la parité des fonctions de Fermi Dirac, on obtient en intégrant par rapport à $k$ l'équation (20) :

$$
\frac{\partial}{\partial t} N(\mu(t, x))+\operatorname{div} j(t, x)=0, \quad \text { avec } \quad j(t, x)=\int_{B} v_{c}(k) h(t, x, k) d k .
$$

ESAIM: Proc., Vol. 11, 2002, 141-152 
D'autre part si $L_{\mu}$ désigne l'opérateur $Q$ linéarisé autour de la fonction de Fermi Dirac on a en identifiant les termes d'ordre $\epsilon$ :

$$
L_{\mu}(h)=v_{c}(k) . \nabla_{x} f(t, x, k)+\nabla_{x} U(t, x) \cdot \nabla_{k} f(t, x, k)
$$

cela permet de calculer $j$ et l'on trouve $j=D(\mu) \nabla_{x}(U+\mu)$ où $D(\mu)$ est une matrice définie positive. En utilisant cette expression dans (23) on obtient l'équation de dérive diffusion :

$$
\frac{\partial}{\partial t} N(\mu(t, x))+\operatorname{div} D(\mu(t, x)) \nabla_{x}(U(t, x)+\mu(t, x))=0 .
$$

Ce passage à la limite peut être totalement justifié, voir [16]. Un des outils essentiel de la démonstration étant les lemmes de compacité en moyenne, [15, 11].

Diverses simplification de (24) permettent alors d'obtenir l'équation classique de Schockley : $\frac{\partial}{\partial t} n+\operatorname{div}(\nu n \nabla U-D \nabla n)=0$ où les coefficients de dérive $\nu$ et de diffusion $D$ sont constants.

Ces équations sont parmi les plus employées en modélisation numérique des semiconducteurs. Cependant leur domaine de validité restreint leur utilisation à des composant de la taille d'une dizaine de micromètres. Or on essaie aujourd'hui d'obtenir au niveau industriel des composants submicroniques. Les équations de dérive diffusion ne peuvent alors plus être employées. Les ingénieurs et les numériciens se sont alors tournés vers des modèles plus complexes, les modèles hydrodynamiques. Ils reposent sur les équations d'Euler avec des termes sources prenant en compte l'effet des collisions et du champ électrique. On peut les obtenir à partir de l'équation de Boltzmann avec des hypothèses ad-hoc sur la fonction de distribution $f$.

Un autre modàle courrament utilisé est le modàle d'énergie transport. Il peut être obtenu par des considérations thermodynamique comme dans le travail de A.M. Anile et O. Muscatto [3] mais aussi par une analyse asymptotique de l'équation de Boltzmann qui a été faite par N. Ben Abdallah, P. Degond et S. Gényeis, [2].

Il existe aussi des modàles intermédiaires où l'énergie est conservée comme variable indépendante : ce sont les modàles S.H.E. (pour Spherical Harmonic Expansion). On pourra se référer à $[2,1,26]$ pour une justification de ces modàles. Le point commun à ces approches est quelles sont basées sur l'hypothàse que les collisions élastiques (celles qui conservent l'énergie) sont prédominantes.

Une autre approche est de considérer des régimes où les phénomànes de dérives dus au champ électrique sont du même ordre de grandeur que les phénomànes collisionnels. Ce sont les approximations sous champ fort de l'équation de Boltzmann qui ont eu un regain d'intérêt récemment, [4, 9, 8, 10, 22].

\section{Références}

[1] N. Ben Abdallah and P. Degond. On a hierarchy of macroscopic models for semiconductors. J. Math. Phys., 37(7):3306-3333, 1996.

[2] N. Ben Abdallah, P. Degond, and S. Genieys. An energy transport model for semiconductors derived from the Boltzmann equation. J. Stat. Phys., 84(1-2):205-231, 1996. 
[3] A. M. Anile and O. Muscato. Improved hydrodynamical model for carrier transport in semiconductors. Phys. Rev. B, 51:16728-16740, 1995.

[4] L. Arlotti and G. Frosali. Runaway particles for a Boltzmann-like transport equation. Math. Models Meth. Appl. Sci., 2(2):203-221, 1992.

[5] P. Bechouche. Semi-classical Limits in a Crystal with a Coulombian self-consistent Potential : Effective Mass Theorems. Asymptotic Analysis, 19:95-116, 1999.

[6] P. Bechouche, N. Mauser, and F. Poupaud. Semi-classical Limits for the Schrödinger Equation in a Crystal with Coulombian Interaction. Manuscript.

[7] P. Bechouche and F. Poupaud. Semi-classical Limits in stratified media. Monatshefte Math., 2000. to appear.

[8] N. Ben Abdallah and H. Chaker. The high field asymptotics for degenerate semiconductors. Math. Models Meth. Appl. Sci. to appear.

[9] N. Ben Abdallah, P. Degond, P.A. Markowich, and C. Schmeiser. High field approximation of the spherical harmonics expansion. Manuscript.

[10] C. Cercignani, I. Gamba, and D. Levermore. High field approximations to a Boltzmann Poisson system and boundary conditions in a semiconductor. Appl. Math. Lett., 10(4):111117, 1997.

[11] R.J. DiPerna and P.L. Lions. Global weak solution of Vlasov-Maxwell systems. Com. on Pure and Appl. Math, XVII :729-757, 1989.

[12] P. Gérard. Mesures semi-classiques et ondes de Bloch. Sem. Ecole Polytechnique, XVI :1-19, 1991.

[13] P. Gérard. Microlocal defect measures. Comm. P.D.E., 16 :1761-1794, 1991.

[14] P. Gérard, P.A. Markowich, N.J. Mauser, and F. Poupaud. Homogenization limits and Wigner transforms. Comm. Pure Appl. Math., L:323-379, 1997.

[15] F. Golse, P.L. Lions, B. Perthame, and R. Sentis. Regularity of the moments of the solution of a transport equation. J. Funct. Anal., 88:110-125, 1988.

[16] F. Golse and F. Poupaud. Limite fluide des équations de Boltzmann des semi-conducteurs pour une statistique de Fermi-Dirac. J. on Asympt. Analysis, 6:135-160, 1992.

[17] P.L. Lions and T. Paul. Sur les mesures de Wigner. Revista Mat. Iberoamericana, 9:553-618, 1993.

[18] P.A. Markowich, N.J. Mauser, and F. Poupaud. A Wigner function approach to semiclassical limits : electrons in a periodic potential. J. Math. Phys., 35:1066-1094, 1994.

ESAIM: Proc., Vol. 11, 2002, 141-152 
[19] P.A. Markowitch, C.A. Ringhofer, and C. Schmeiser. Semiconductors Equations. Springer, Wien-New York, 1989.

[20] A. Nouri and F. Poupaud. Stationary solutions of boundary value problems for Maxwell Boltzmann system modeling degenerate semiconductors. SIAM J. Math. Anal., 26:1143$1156,1995$.

[21] F. Poupaud. On a system of non linear Boltzmann equations of semiconductor physics. SIAM J. on Appl. Math., 50:1593-1606, 1990.

[22] F. Poupaud. Runaway phenomena and fluid approximation under high fields in semiconductor kinetic theory. ZAMM, 72:359-372, 1992.

[23] F. Poupaud and C. Ringhofer. Semi-classical limits in a crystal with external potentials and effective mass theorems. Com. Part. Dif. Eq., 21:1897-1918, 1996.

[24] M. Reed and B. Simon. Methods of Modern Mathematical Physics IV. Academic Press, New York-San Francisco-London, 4th edition, 1987.

[25] L. Tartar. H-measures: a new approach for studying homogenization, oscillations and concentration effects in partial differential equations. Proc. Roy. Soc. Edinburgh Sect. A, 1990.

[26] D. Ventura, A. Gnudi, G. Baccarani, and Odeh F. Multidimensional spherical harmonics expansion of Boltzmann equation for transport in semiconductors. Appl. Math. Lett., 5:8590, 1992.

[27] E. Wigner. On the quantum correction for thermodynamic equilibrium. Phys. Rev., 40:749759, 1932.

[28] C.H. Wilcox. Theory of Bloch waves. J. d'Analyse Math., 33:146-167, 1978.

Frédéric Poupaud

Laboratoire J.A. Dieudonné (UMR CNRS 6621)

Université de Nice

Parc Valrose

BP 71

F06108 Nice Cedex 02, France

mailto:poupaud@math.unice.fr 